Internat. J. Math. \& Math. Sci.

Vol. 23, No. 9 (2000) 645-649

S0161171200002489

(C) Hindawi Publishing Corp.

\title{
ON THE DEGREE OF APPROXIMATION BY GAUSS WEIERSTRASS INTEGRALS
}

\section{HUZOOR H. KHAN and GOVIND RAM}

(Received 1 July 1998 and in revised form 15 October 1998)

\begin{abstract}
We obtain the degree of approximation of functions belonging to class $\operatorname{Lip}(\psi(u, v) ; p), p>1$ using the Gauss Weierstrass integral of the double Fourier series of $f(x, y)$.
\end{abstract}

Keywords and phrases. Lip $(\psi(u, v) ; p)$ class, Gauss Weierstrass integral, arithmetic means, Jackson operator, degree of approximation, Fourier series, Hölder's inequality.

2000 Mathematics Subject Classification. Primary 41A25.

1. Introduction and results. Let the function $f(x, y)$ be integrable in the sense of Lebesgue over the square $(0,2 \pi ; 0,2 \pi)$ and periodic with period $2 \pi$ in each variable outside the square. Let the double Fourier series of $f(x, y)$ be

$$
\sum_{m=0}^{\infty} \sum_{n=0}^{\infty} A_{m, n}(x, y)
$$

where

$$
\begin{gathered}
A_{0,0}(x, y)=\frac{1}{4} a_{0,0}, \\
A_{m, 0}(x, y)=\frac{1}{2}\left(a_{m, 0} \cos m x+b_{m, 0} \sin m x\right), \\
A_{0, n}(x, y)=\frac{1}{2}\left(a_{0, n} \cos n x+b_{0, n} \sin n x\right), \\
A_{m, n}(x, y)=a_{m, n} \cos m x \cos n y+b_{m, n} \cos m x \sin n y \\
+c_{m, n} \sin m x \cos n y+d_{m, n} \sin m x \sin n y .
\end{gathered}
$$

Let

$$
\begin{aligned}
\phi(u, v)=\frac{1}{4}\{ & f(x+u, y+v)+f(x-u, y+v)+f(x+u, y-v) \\
+ & f(x-u, y-v)-4 f(x, y)\}
\end{aligned}
$$

The series

$$
\sum_{m=0}^{\infty} \sum_{n=0}^{\infty} \lambda_{m, n} A_{m, n}(x, y)
$$

is called the double Fourier series associated with the function $\phi(u, v)$ such that 


$$
\lambda_{m, n}= \begin{cases}\frac{1}{4} & \text { for } m=0, n=0, \\ \frac{1}{2} & \text { for } m=0, n>0 ; m>0, n=0, \\ 1 & \text { for } m>0, n>0 .\end{cases}
$$

Also, the coefficients in the series (1.4) are given by

$$
a_{m, n}=\frac{1}{\pi^{2}} \int_{0}^{2 \pi} \int_{0}^{2 \pi} \phi(u, v) \cos m u \cos n v d u d v
$$

and three other similar expressions defining $b_{m, n}, c_{m, n}, d_{m, n}$.

We define the Gauss Weierstrass integral of $f(x, y)$ by

$$
\begin{aligned}
W_{m, n}(x, y) & =W(x, y ; \xi, \eta)=\sum_{k=0}^{\infty} \sum_{\ell=0}^{\infty} \frac{1}{\exp \left(\left(k^{2} \xi / 4\right)+\left(\ell^{2} \eta / 4\right)\right)} A_{k, \ell}(x, y) \\
& =\sqrt{\frac{\pi}{(\xi, \eta)}} \int_{-\pi}^{\pi} \int_{-\pi}^{\pi} \frac{f(x+t, y+s)}{\exp \left(\left(t^{2} / \xi\right)+\left(s^{2} / \eta\right)\right)} d t d s+o(\xi, \eta),
\end{aligned}
$$

where $o(\xi, \eta) \rightarrow 0$ as $\xi \rightarrow 0, \eta \rightarrow 0$, and $(\xi, \eta)$ is the product of $\xi$ and $\eta$.

A $2 \pi$ periodic function $f(x, y)$ in each variable $x$ and $y$ is said to belong to the class $\operatorname{Lip}(\psi(u, v) ; p), p>1$, [2] if

$$
|f(x+u, y+v)-f(x, y)| \leq M\left(\frac{\psi(u, v)}{(u, v)^{1 / p}}\right), \quad 0<u<\pi, 0<v<\pi,
$$

where $\psi(u, v)$ is a positive increasing function of the variables $u, v$ and $M$ is a positive number independent of $x, u$ and $v$.

Yoshimitsu, [1] proved a theorem for obtaining the degree of approximation of class of functions $\operatorname{Lip}(\alpha, \beta), 0<\alpha<1$ and $0<\beta<1$, by means of the first arithmetic means of double Fourier series. Siddiqi and Mohammadzadeh [3] extended the result in two directions in terms of a positive increasing function of two variables. Recently Khan, [2] extended the result of Yoshimitsu, Siddiqi et al. for the more general operator, the Jackson type operator, and more general class of functions, $\operatorname{Lip}(\psi(u, v) ; p), p>1$. The object of the present paper is to determine the degree of approximation for the functions belonging to the class $\operatorname{Lip}(\psi(u, v) ; p), p>1$, by means of Gauss Weierstrass integral of the double Fourier series of $f(x, y)$.

Our theorem states as follows.

THEOREM 1.1. Let $f(x, y)$ be a continuous function of period $2 \pi$ with respect to each variable $x$ and $y$ belonging to $\operatorname{Lip}(\psi(u, v) ; p), p>1$ class, then

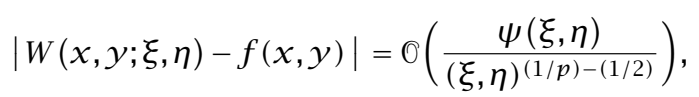

provided

$$
\left[\int_{0}^{\xi} \int_{0}^{\eta}\left(\frac{\psi(t, s)}{(t, s)^{1 / p}}\right)^{p} d t d s\right]^{1 / p}=\mathcal{O}(\psi(\xi, \eta))
$$




$$
\left[\int_{0}^{\xi} \int_{\eta}^{\pi}\left(\frac{\psi(t, s)}{(t, s)^{2+(1 / p)}}\right)^{p} d t d s\right]^{1 / p}=O\left(\frac{\psi(\xi, \eta)}{(\xi, \eta)^{2}}\right) .
$$

2. Proof of the theorem. Using (1.7), we get

$$
\begin{aligned}
W(x, y ; \xi, \eta)-f(x, y) & \\
& =\sqrt{\frac{\pi}{(\xi, \eta)}} \int_{-\pi}^{\pi} \int_{-\pi}^{\pi} \phi(t, s) e^{-\left(\left(t^{2} / \xi\right)+\left(s^{2} / \eta\right)\right)} d t d s+R(x, y ; \xi, \eta) \\
& =4 \sqrt{\frac{\pi}{(\xi, \eta)}} \int_{0}^{\pi} \int_{0}^{\pi} \phi(t, s) e^{-\left(\left(t^{2} / \xi\right)+\left(s^{2} / \eta\right)\right)} d t d s+\mathcal{O}(\xi, \eta) \\
& =4 \sqrt{\frac{\pi}{(\xi, \eta)}}\left(\int_{0}^{\xi} \int_{0}^{\eta}+\int_{0}^{\xi} \int_{\eta}^{\pi}+\int_{\xi}^{\pi} \int_{0}^{\eta}+\int_{\xi}^{\pi} \int_{\eta}^{\pi}\right) \phi(t, s) e^{-\left(\left(t^{2} / \xi\right)+\left(s^{2} / \eta\right)\right)} d t d s \\
& =I_{1,2}(x, y)+I_{1,3}(x, y)+I_{4,2}(x, y)+I_{4,3}(x, y) .
\end{aligned}
$$

Applying Hölder's inequality of two variables and the fact that $\phi(u, v) \in \operatorname{Lip}(\psi(u$, $v) ; p), p>1$ for $I_{1,2}(x, y)$, we get

$$
\begin{aligned}
\left|I_{1,2}(x, y)\right| \leq & 4 \sqrt{\frac{\pi}{(\xi, \eta)}}\left[\int_{0}^{\xi} \int_{0}^{\eta}|\phi(t, s)|^{p} d t d s\right]^{1 / p} \\
\cdot & {\left[\int_{0}^{\xi} \int_{0}^{\eta}\left(e^{-\left(\left(t^{2} / \xi\right)+\left(s^{2} / \eta\right)\right)}\right)^{p^{\prime}} d t d s\right]^{1 / p^{\prime}}, }
\end{aligned}
$$

where

$$
\begin{aligned}
\frac{1}{p^{\prime}} & =\frac{p-1}{p} \\
& \leq 4 \sqrt{\frac{\pi}{(\xi, \eta)}}\left[\int_{0}^{\xi} \int_{0}^{\eta}\left(\frac{\psi(t, s)}{(t, s)^{1 / p}}\right)^{p} d t d s\right]^{1 / p}\left[\int _ { 0 } ^ { \xi } \int _ { 0 } ^ { \eta } \left(e^{\left.\left.-\left(t^{2} / \xi\right)+\left(s^{2} / \eta\right)\right)^{p^{\prime}} d t d s\right]^{1 / p^{\prime}}}\right.\right. \\
& =\mathcal{O}\left(\frac{\psi(\xi, \eta)}{(\sqrt{\xi}, \sqrt{\eta})}\right)\left(\int_{0}^{\xi} \int_{0}^{\eta} e^{-\left(t^{2} p^{\prime / \xi}\right)} e^{-\left(s^{2} p^{\prime / \eta}\right)} d t d s\right)^{1 / p^{\prime}} \text { using condition (1.10) } \\
& =\mathcal{O}\left(\frac{\psi(\xi, \eta)}{(\sqrt{\xi}, \sqrt{\eta})}\right)\left(\int_{0}^{\sqrt{\xi p^{\prime}}} e^{-u^{2}} \frac{\sqrt{\xi}}{\sqrt{p^{\prime}}} d u \int_{0}^{\sqrt{\eta p^{\prime}}} e^{-v^{2}} \frac{\sqrt{\eta}}{\sqrt{p^{\prime}}} d v\right)^{1 / p^{\prime}} \\
& =\mathcal{O}\left(\frac{\psi(\xi, \eta)}{(\sqrt{\xi}, \sqrt{\eta})}\right) \mathcal{O}(\xi, \eta)^{1 / p^{\prime}}=\mathcal{O}\left(\frac{\psi(\xi, \eta)}{(\xi, \eta)^{(1 / p)-(1 / 2)}}\right)
\end{aligned}
$$

where $u=t \sqrt{p^{\prime} / \xi}$ and $v=s \sqrt{p^{\prime} / \eta}$.

Applying Hölder's inequality of two variables to $I_{1,3}(x, y)$ and using the fact that $\phi(u, v) \in \operatorname{Lip}(\psi(u, v) ; p), p>1$, we get 


$$
\begin{aligned}
\left|I_{1,3}(x, y)\right| \leq & \frac{4 \sqrt{\pi}}{(\sqrt{\xi}, \sqrt{\eta})} \\
& \cdot\left[\left(\int_{0}^{\xi} \int_{\eta}^{\pi}\left|\frac{\phi(t, s)}{(t, s)^{2}}\right|^{p} d t d s\right)^{1 / p}\left(\int_{0}^{\xi} \int_{\eta}^{\pi} \frac{e^{-\left(t^{2} / \xi\right)+\left(s^{2} / \eta\right) p^{\prime}}}{(t, s)^{-2 p^{\prime}}} d t d s\right)^{1 / p^{\prime}}\right] \\
\leq & \frac{4 \sqrt{\pi}}{(\sqrt{\xi}, \sqrt{\eta})}\left\{\left[\int_{0}^{\xi} \int_{\eta}^{\pi}\left(\frac{\psi(t, s)}{(t, s)^{2+(1 / p)}}\right)^{p} d t d s\right]^{1 / p}\right. \\
& \left.\cdot\left(\int_{0}^{\xi} e^{-t^{2} p^{\prime} / \xi} \cdot t^{2 p^{\prime}} d t \int_{\eta}^{\pi} e^{-s^{2} p^{\prime} / \eta} s^{2 p^{\prime}} d s\right)^{1 / p^{\prime}}\right\} \\
= & \mathbb{O}\left(\frac{\psi(\xi, \eta)}{(\xi, \eta)^{5 / 2}}\right)\left\{\left[\int_{0}^{\sqrt{\xi p^{\prime}}} e^{-u^{2}}\left(\frac{\sqrt{\xi}}{\sqrt{p}} u\right)^{2 p}\left(\frac{\sqrt{\xi}}{\sqrt{p}}\right) d u\right]^{1 / p}\right. \\
& \left.\cdot\left[\int_{\sqrt{\eta p^{\prime}}}^{\left(\pi / \sqrt{\left.\eta p^{\prime}\right)}\right.} e^{-v^{2}}\left(\frac{\sqrt{\eta}}{\sqrt{p^{\prime}}} v\right)^{2 p^{\prime}}\left(\frac{\sqrt{\eta}}{\sqrt{p^{\prime}}}\right) d v\right]\right\} \\
= & \mathbb{O}\left(\frac{\psi(\xi, \eta)}{(\xi, \eta)^{5 / 2}}\right) \mathcal{O}(\xi, \eta)^{2+\left(1 / p^{\prime}\right)}=\mathcal{O}\left(\frac{\psi(\xi, \eta)}{(\xi, \eta)^{(1 / p)-(1 / 2)}}\right) .
\end{aligned}
$$

Similarly, we can prove that

$$
\left|I_{4,2}(x, y)\right|=\mathcal{O}\left(\frac{\psi(\xi, \eta)}{(\xi, \eta)^{(1 / p)-(1 / 2)}}\right), \quad\left|I_{4,3}(x, y)\right|=\mathcal{O}\left(\frac{\psi(\xi, \eta)}{(\xi, \eta)^{(1 / p)-(1 / 2)}}\right) .
$$

Finally, we get

$$
W(x, y ; \xi, \eta)-f(x, y)=\mathcal{O}\left(\frac{\psi(\xi, \eta)}{(\xi, \eta)^{(1 / p)-(1 / 2)}}\right) .
$$

REMARK 2.1. It may also be remarked that by giving different values to $\psi(u, v)$, we get some interesting results:

(i) If $\psi(u, v)=u^{\alpha} * v^{\beta}$, [1], $0<\alpha<1,0<\beta<1$, then we have

$$
|W(x, y ; \xi, \eta)-f(x, y)|=\mathcal{O}\left(\xi^{\alpha+(1 / 2)-(1 / p)} * \eta^{\beta+(1 / 2)-(1 / p)}\right) .
$$

(ii) If $\psi(u, v)=J(u, v)(u, v)^{1 / p}$, [3], where $J(u, v)$ is a positive increasing function of variables $u$ and $v$, then

$$
|W(x, y ; \xi, \eta)-f(x, y)|=\mathcal{O}\left(\frac{J(\xi, \eta)}{(\xi, \eta)^{-1 / 2}}\right) .
$$

\section{REFERENCES}

[1] Yoshimitsu H., On summabilities of double Fourier series, Kōdai Math. Sem. Rep. 15 (1963), 226-238. MR 28\#5299. Zbl 127.29302.

[2] Huzoor H. Khan, On the degree of approximation by Jackson type operators, Preprint of International Centre for Theoretical Physics, Trieste, Itlay (October 1994). IC/94/344. 
[3] A. H. Siddiqui and M. Mohammadzadeh, Approximation by Cesáro and B means of double Fourier series, Math. Japon. 21 (1976), no. 4, 343-349. MR 56\#977. Zbl 367.40003.

Khan and Ram: Department of Mathematics, Aligarh Muslim University, Aligarh202 002, INDIA 


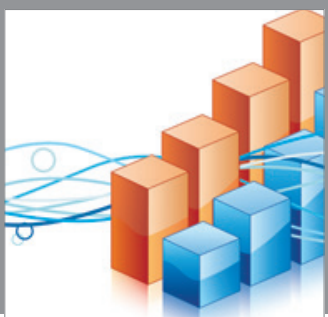

Advances in

Operations Research

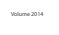

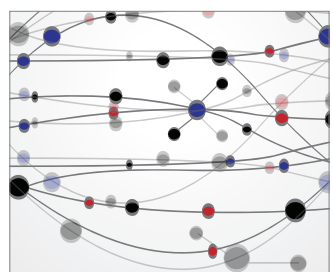

\section{The Scientific} World Journal
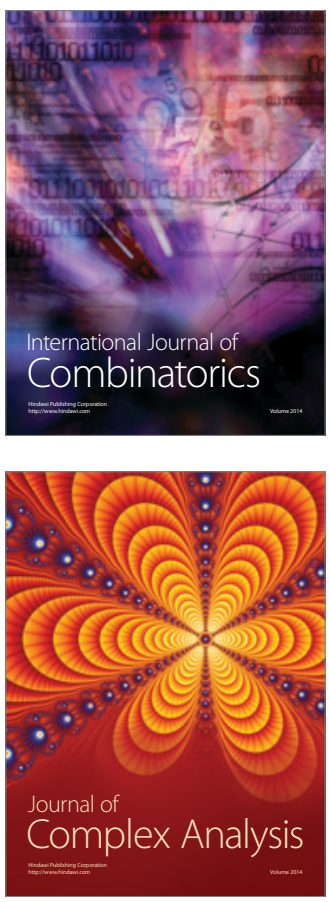

International Journal of

Mathematics and

Mathematical

Sciences


Journal of

Applied Mathematics
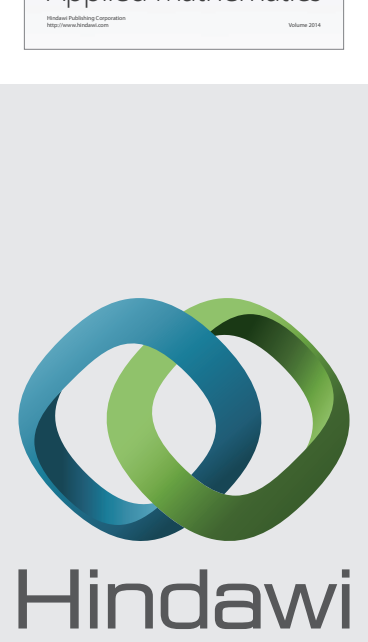

Submit your manuscripts at http://www.hindawi.com
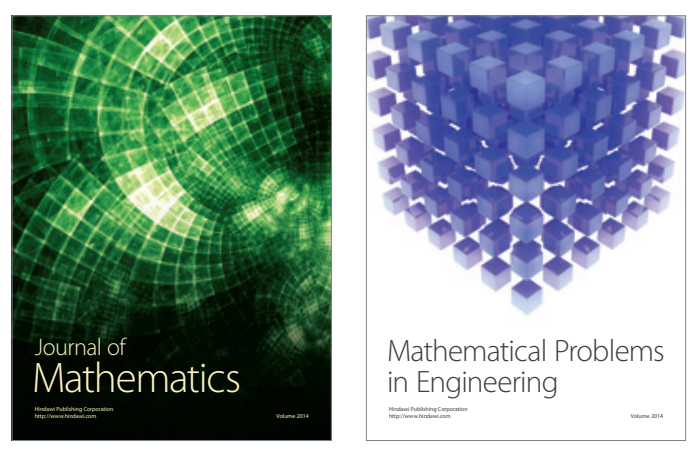

Mathematical Problems in Engineering
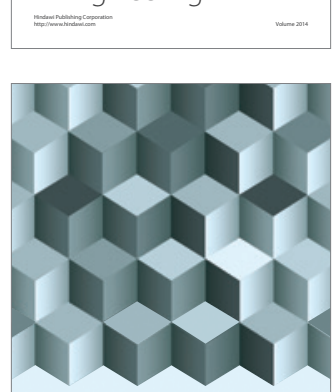

Journal of

Function Spaces
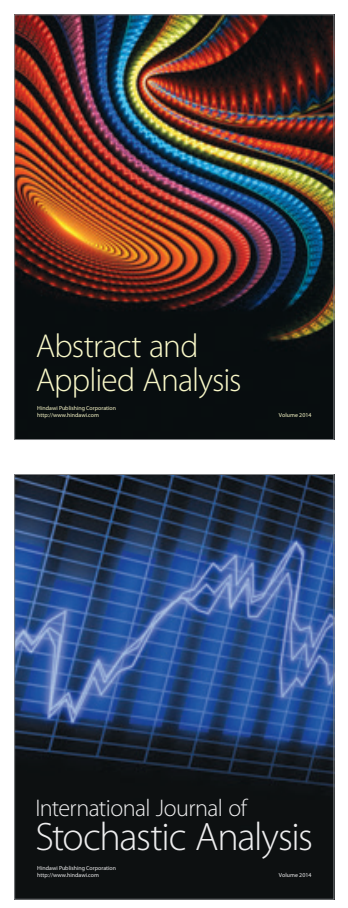



ournal of

Probability and Statistics

Promensencen
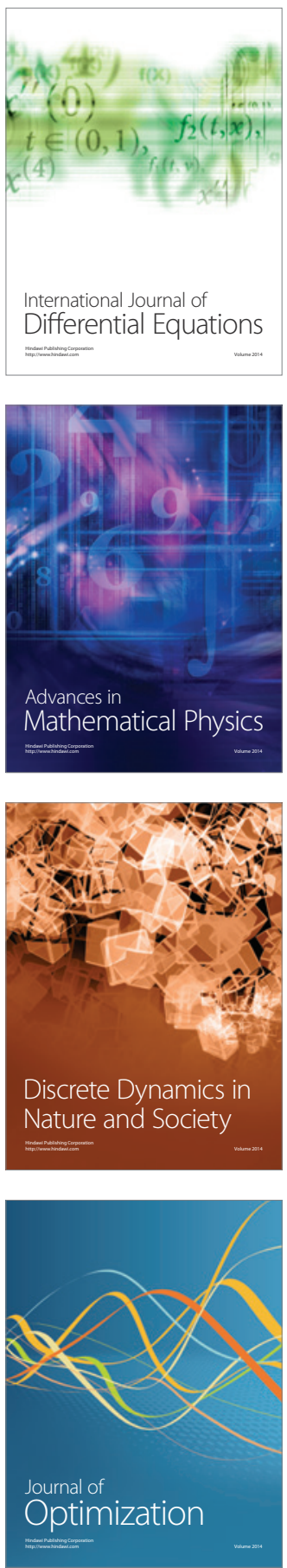